\title{
Polarization-sensitive optical coherence tomography for ceramic materials inspection
}

\author{
M. Trojanowski, ${ }^{*}$ M. Kraszewski, M. Strąkowski, B.B. Kosmowski, and J. Pluciński \\ Department of Metrology and Optoelectronics, Faculty of Electronics Telecommunications and Informatics, \\ Gdańsk University of Technology, Narutowicza 11/12, 80-233 Gdańsk
}

Received December 08, 2014; accepted December 24, 2014; published December 31, 2014

\begin{abstract}
Ceramics production is looking for a fast, reliable and non-destructive method that can be implemented on site for defect detection and analysis. In this paper we present polarization-sensitive optical coherence tomography (PS-OCT) as a method for defect inspection. The proposed extensions to standard OCT provide additional information for complete characterization of a tested object. We compare OCT and microscope imaging that can easily detect enamel layer defects. The object under test is a sample ceramic plate with several defects occurring in production process. The results of OCT imaging show those defects are only in the enamel layer without any effect on the potsherd layer.
\end{abstract}

In ceramic tableware production there is a great need for creating uniform and high quality products. Maintaining such quality is difficult if occurring defects are not diagnosed correctly. The most useful methods are based on visual observation of the ceramics surface. They provide enough information to assess the quality of the end product, however, the defects which are under the cover layer are mostly left out. The main problems are occurring ceramic layer gaps and ceramics inhomogeneity, which are of greatest concern. These issues might have a significant influence on products future strength in normal use. Moreover, some of these defects come from the components of the potsherd at the beginning of the manufacturing process. Very often they become the cause of surface defects, the reason of which the end products must be rejected. Therefore, a fast, reliable and effective method for subsurface defect and unwanted inclusion detection would improve the quality of finished ceramic products and reduce the cost of their manufacturing. To address this problem, we propose the usage of optical coherence tomography (OCT) with additional polarization state detection (PS-OCT) [1-2]. OCT has many implementations for medical diagnosis and for technical object inspection [3-8]. It was used with great success to analyse a wide range of technical objects due to its high resolution and possibility of subsurface imaging [9]. Various researches were also performed on OCT application for ceramics and composite materials quality assessment [2, 10-14].

\footnotetext{
*E-mail: mictroja@pg.gda.pl
}

One of the most valuable features of OCT is that it is a non-destructive, non-contact and non-invasive imaging method that gives tomographic images of inner structure [15]. In order to obtain such images, OCT uses white light interferometry, which allows to gather depth related information from scattering objects inner structure. Standard realization of OCT gives intensity images, which express information about the intensity of the backscattered light from the tested device. It means that by the use of a standard OCT system one can observe high and low scattering areas which introduce intensity changes in the tomographic image. Polarization state information about backscattered light can be also helpful in detecting full defects and their characterization. In the future an additional spectroscopic analysis will be used to characterize ceramic layer interruptions better [16].

In this paper, on a sample plate we demonstrate the usefulness of PS-OCT for the analysis of defects in ceramic layers. The number of different types of defects, which mainly occur in ceramics, were presented and OCT measurements have been shown.

As a device under test, a white ceramic plate with several defects in its ceramic layers has been chosen. These defects were made during the manufacturing process and can be seen as small spots, pits. On the whole surface, there are two major groups with defects that have been taken into consideration. The ceramics, also called potsherd, is covered by a transparent enamel layer. One of the very important issues is defect localization. Standard microscope tools and OCT system were used to find out on which layer, potsherd or enamel, the defect occurs.

PS-OCT system (see Fig. 1), which was applied for the investigation, works with a central wavelength of 1320 $\mathrm{nm}$ and a spectral range of $120 \mathrm{~nm}$. It corresponds to an axial resolution of $12 \mu \mathrm{m}$ and lateral resolution of $15 \mu \mathrm{m}$. The system allows to analyse the polarization state of the light backscattered from the examined object. The object is illuminated by circularly polarized light (right-handed) and the detection is separated for two orthogonal states of circular light polarization: right- and left-handed. The 
polarization of the detected light is visualized as a "retardation angle" defined as [14]:

$$
\gamma=\arctan \sqrt{\frac{I_{R H}}{I_{L H}}}
$$

where $I_{R H}$ and $I_{L H}$ are the intensities of light with rightand left-handed polarizations accordingly.

With the aid of this system, we can visualize the inner structure of enamel layer interruptions and, in addition, we can gather retardation angle information. This system is equipped with dedicated software that allows OCT intensity measurements and polarization sensitive analysis to be visualized together in the same OCT image.

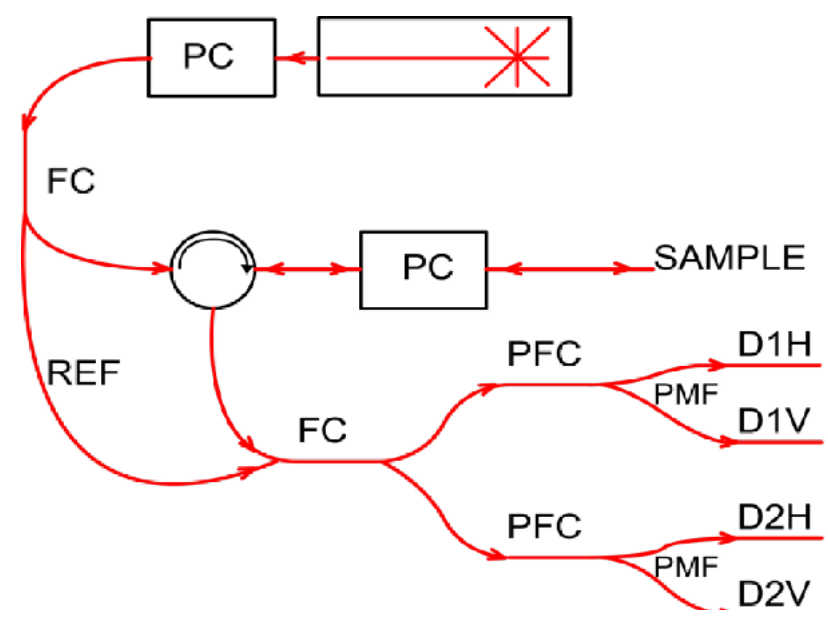

Fig. 1. PS-OCT system scheme (PC - polarization control, FC - fiber coupler, $\mathrm{PFC}$ - polarization fiber coupler, $\mathrm{PMF}$ - polarization maintaining fiber, $\mathrm{D} 1 \mathrm{H}$ - detector 1 , horizontal polarization, D1V detector 1 , vertical polarization, REF - reference arm).

As a reference measurement, we have used an NJF120A metallurgical microscope that allowed to localize and estimate the size and shape of defects. Images were taken with a magnification of 64 .

Two defects from separate parts of the plate were compared in order to identify the type of occurring inhomogeneities in the ceramic layer. Firstly, to localize and identify defects, we used a metallurgical microscope (Figs. 2 and 3).

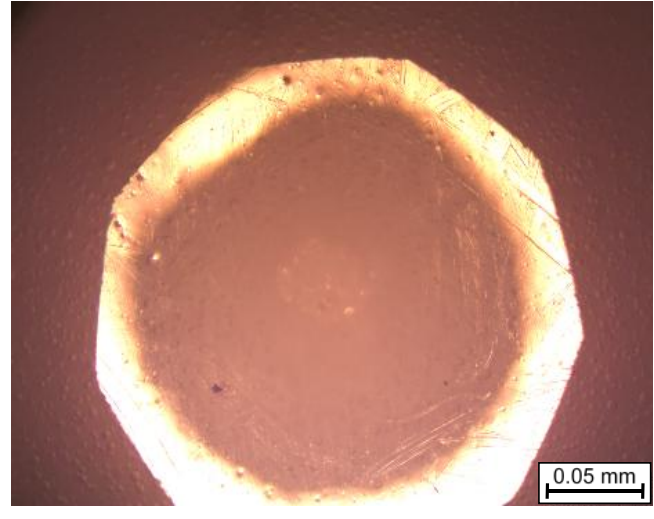

Fig. 2. Microscope image of defected area from a first point of interest $(0.21 \mathrm{~mm}$ size of defect)

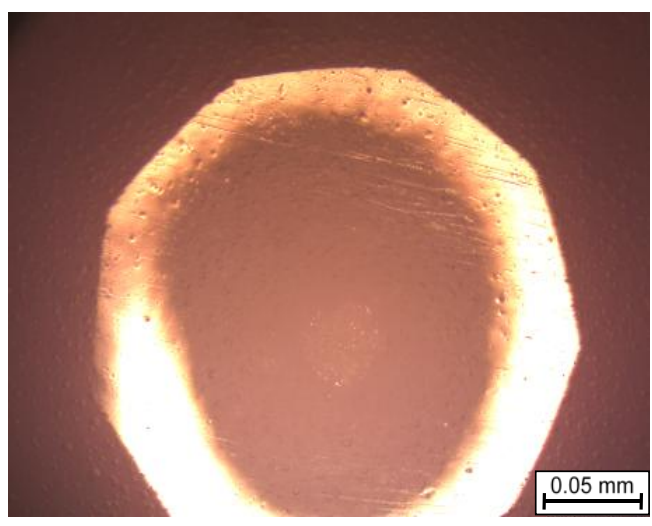

Fig. 3. Microscope image of defect from a second area of interest $(0.17$ $\mathrm{mm}$ size of defect).

The application of the microscope allowed to evaluate the size and shape of inhomogeneities and observe them at the enamel level. For better inspection of the defected area, complementary measurements based on the OCT technique were carried out. In order to measure the depth and exact size of defects, OCT has been used (Fig. 4).

The depth was measured from the top of the enamel layer to the potsherd layer. For both defects the diameter was measured at their half height. The results are as shown in Table 1.

Table 1. Diameter and height of defects measured with OCT.

\begin{tabular}{|c|c|c|}
\hline & Defect a) & Defect b) \\
\hline Diameter [mm] & 0.93 & 0.59 \\
\hline Depth [mm] & 0.08 & 0.04 \\
\hline
\end{tabular}



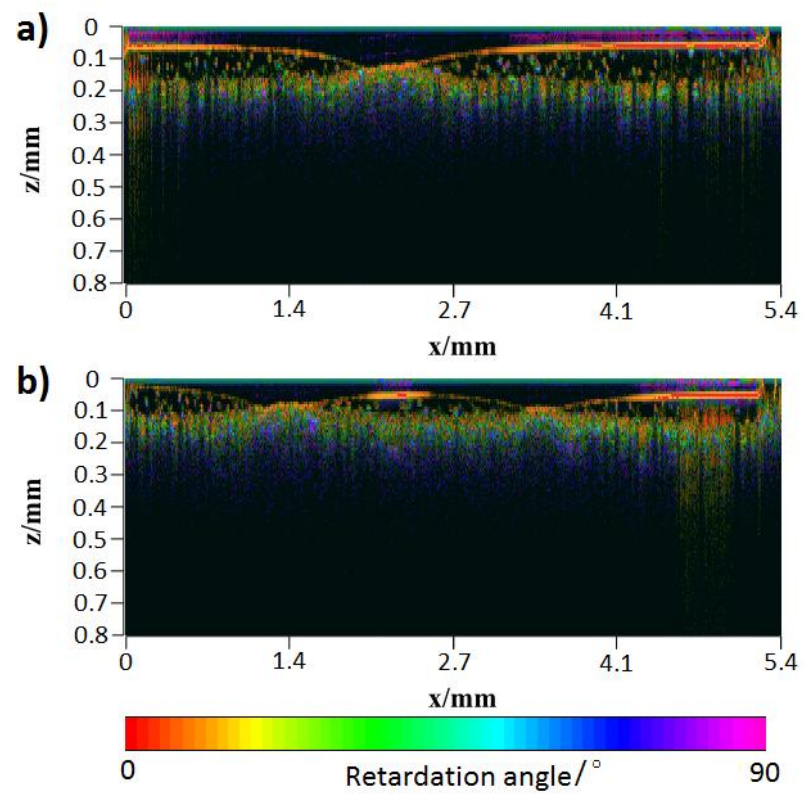

Fig. 4. Cross-sectional images of the defects; a) OCT image taken for defect 1 (Fig. 2 - microscope image), b) OCT image taken for defect 2 (Fig. 3 - microscope image).

In this work, we have presented the problem of non-destructive inspection of defects occurring in ceramic tableware. The first type of measurements (microscope and eye inspection) is capable of giving information about the shape of a defect. It can be easily used to localize defects. However, this method suffers from an inability to localize the defective layer and determine quantitative parameters. Moreover, by using the visual inspection and microscope measurements it is difficult to determine the character and type of the defects, as well as their localization inside the enamel or potsherd layer. This problem requires the usage of tomographic imaging techniques such as OCT, which allows to see under the surface of enamel and gives additional information about the defect. OCT, which was used as a main research method, clearly shows that defects occur only in the enamel layer without any visual effect on the potsherd layer. According to OCT measurements, the enamel layer vanishes in the defected area. For evaluating the potsherd layer, polarization analysis is very useful due to random retardation angle changes from a homogenous potsherd layer and constant retardation angle changes for an enamel layer. This allows to identify if there are any inclusions in the potsherd layer that may lead to any future defects. That kind of information can correspond to inventing new methods of production supervision that will eliminate any type of defects. In addition, OCT measurements can easily be automated and implemented in current production lines giving on site evaluation of the production process.
In conclusion, the research work presented in this paper shows that with an application of OCT and additional retardation angle analysis one can detect and identify defects occurring in ceramics. The tested piece of tableware had several inhomogeneities in its enamel layer, which have been successfully detected and measured (Fig. 4). In addition, the results show that those defects affected only the enamel layer without any changes in the potsherd. Further analysis of ceramics will be conducted and spectroscopic analysis will be used to identify the defects of a potsherd.

This research work has been supported by The National Centre of Research and Development (NCBiR), Poland under grant no. LIDER/32/205/L-3/11 and DS program of Faculty of Electronics, Telecommunications and Informatics, Gdańsk University of Technology.

\section{References}

[1] M.R. Strąkowski, J. Pluciński, B.B. Kosmowski, Phot. Lett. Poland 3, 128 (2011).

[2] W. Drexler, J.G. Fujimoto, Optical Coherence Tomography. Technology and Applications (Springer, 2008).

[3] M. Bashkansky, M.D. Duncan, J. Reintjes, AIP Conf. 509, 1517 (2000).

[4] J.P. Dunkers, F.R. Phelan, D.P. Sanders, M.J. Everett, W.H. Green, D.L. Hunston, R.S. Parnas, Opt. and Lasers in Eng. 35 (2001).

[5] R. Su, E. Chang, P. Ekberg, L. Mattsson, S. Hyun Yun, $1^{\text {st }}$ International Symposium on OCT4NDT, Linz (2013).

[6] M. Baskansky, M.D. Duncan, M. Kahn, D. Lewis III, J. Reintjes, Opt. Lett. 22, 61-63 (1997).

[7] K. Wiesauera, M. Pircherb, E.G. Otzingerb et al., Opt. Expr. 13(3), 1015-1025 (2005).

[8] G. Song, K. Harding, Proc. SPIE 8563, 85630N (2012).

[9] B. Saleh, Introduction to subsurface imaging (Cambridge University Press, 2011).

[10] R. Su, M. Kirillin, P. Ekberg, A. Roos, E. Sergeeva, L. Mattsson, Opt. Expr. 20, 4603 (2012).

[11] M. Bashkansky, M.D. Duncan, M. Kahn, D. Lewis Iii, J. Reintjes, Opt. Lett. 22, 61 (1997).

[12] A.K.S. Braz, B.B.C. Kyotoku, R. Braz, A.S.L. Gomes, Dent. Mater 25, 74 (2009). doi: 10.1016/j.dental.2008.04.011.

[13] C. Sinescu, M.L. Negrutiu, C. Ionita, F. Topala, E. Petrescu, R. Rominu, D.M. Pop, L. Marsavina, R. Negru, A. Bradu, M. Rominu, A.G. Podoleanu, Proc. SPIE 7990, 79900W (2010). doi: $10.1117 / 12.890272$

[14] A.F. Fercher, Reports on Progr. Phys. 66, 239 (2003).

[15] D. Stifter, A.D. Sanchis Dufau, E. Breuer, K. Wiesauer, P. Burgholzer, O. Höglinger, E. Götzinger, M. Pircher, C.K. Hitzenberger, Insight 47, 209 (2005).

[16] M.R. Strąkowski, M. Kraszewski, M. Trojanowski, J. Pluciński, Proc. SPIE 9132, 91320N (2014). 